\title{
A Miniature Robot for Autonomous Single Neuron Recordings
}

\author{
Edward A. Branchaud, Jorge G. Cham, Zoran Nenadic, \\ and Joel W. Burdick \\ Division of Engineering and Applied Science \\ California Institute of Technology \\ Pasadena, CA 91125 \\ eddie@caltech.edu
}

\author{
Richard A. Andersen \\ Division of Biology \\ California Institute of Technology \\ Pasadena, CA 91125
}

\begin{abstract}
This paper describes a novel miniature robot that can autonomously position recording electrodes inside cortical tissue to isolate and maintain optimal extracellular action potential recordings. The system consists of a novel motorized miniature recording microdrive and a control algorithm. The microdrive was designed for semi-chronic operation and can independently position four electrodes with micron precision over a $5 \mathrm{~mm}$ range using small $(3 \mathrm{~mm}$ diameter) piezoelectric linear actuators. The autonomous positioning algorithm is designed to detect, align and cluster action potentials, and then command the microdrive to optimize and maintain the neural signal. This system is shown to be capable of autonomous operation in monkey cortex.
\end{abstract} Robot.

Index Terms - Medical Robotics, Neurorobotics, Miniature

\section{INTRODUCTION}

This paper describes a novel miniature robot and its associated control algorithm. This system is aimed at improving the ability of neuroscientists and clinical doctors to record high quality extracellular signals in neuronal tissue. Before describing this device and our experimental results, we first very briefly review the current practice of extracellular recording of neurons.

\section{A. Current Issues in Extracellular Recording}

Information transfer and processing in the brain occurs through the transmission of electrical pulses, called action potentials, between neurons. Studying the patterns of action potentials associated with individual neurons while a subject (e.g. a rat, fly, monkey, or human) is presented with a stimulus or engages in a behavioral task is a principal tool for studying brain areas. Noninvasive methods such as fMRI or EEG recordings can provide gross estimates of activity levels in a given region, but recording action potentials of individual neurons is necessary to understand how information is processed in local neural networks.

Recordings of action potentials are made by inserting electrodes (typically sharpened metal wires insulated along their length and exposed at the tip) into the neural tissue. There are two dominant modes of recordings. In acute recordings, electrodes are inserted and removed from the neural tissue each recording session. In chronic recordings, electrodes are surgically implanted and remain in place for weeks or months at a time.
For acute recordings, a portion of the skull over the brain region of interest is removed and replaced with a sealable chamber. During a recording session, a device termed a microdrive is affixed to the opened chamber and is used to advance the electrodes into the neural tissue, usually in a motorized fashion. The electrode motion is controlled by the experimenter until the quality of the action potential signals is acceptable. This process is commonly guided by experience, intuition and feedback from visual and auditory representations of the voltage signal. The electrode is positioned close enough to the neuron for a high quality recording, yet far enough away to avoid damaging it. The electrode must be periodically repositioned to maintain the signal as the tissue that was compressed during the electrode insertion process relaxes, causing significant signal drift. The process of finding and holding neural signals consumes a significant amount of the experimenter's time and focus. As the number of electrodes increases (commercial microdrives with up to sixteen electrodes are currently available), the task of continuously positioning each electrode to maintain high quality neural signal becomes intractable for a single experimenter.

In chronic recordings, stationary multi-electrode assemblies, which are typically bundles or arrays of thin wires or silicon probes, are surgically implanted in the region of interest [1-3]. The signal yield of the implant array, i.e. the percentage of the array's electrodes that record active cells, depends upon the luck of the initial surgical placement. The electrodes may be placed in inactive tissue, or the

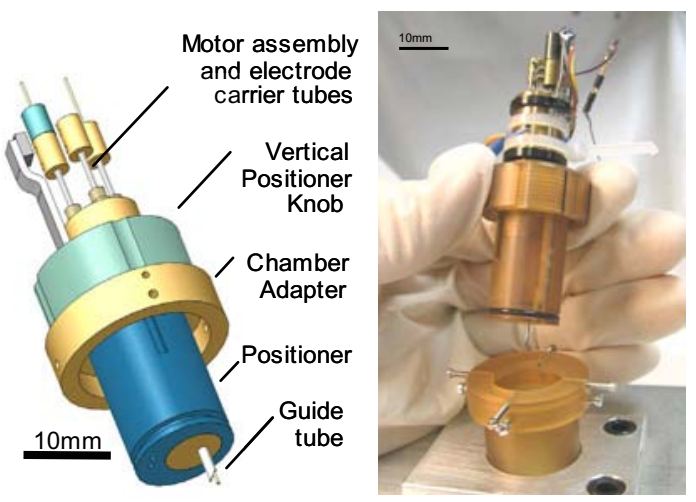

Figure 1. Prototype miniature robot with movable electrodes. The device is capable of positioning four electrodes to optimize recordings of action potentials.

\footnotetext{
* This work was supported by DARPA Grant MDA972-00-1-0029 and by the Center for Neuromorphic Engineering, an NSF Engineering Research Center (NSF-9402726).
} 
wrong brain region. Even if properly placed, the active recording site may not sit sufficiently close to an active cell body (the listening sphere of a neuron is typically 50-100 microns [4]). Moreover, even if the electrode is initially well placed, tissue migrations and local tissue reactions can cause subsequent loss of signal, thereby reducing or disabling the function of the recording array over time.

A chronic implant in which the electrodes can be continually repositioned after implantation could overcome these limitations and greatly extend the signal yield and lifetime of chronic array implants. In existing chronic microdrives, the electrodes are manually repositioned either by turning lead screws or by affixing a conventional microdrive to the array [5-13]. As the number of electrodes increases, this manual repositioning becomes impractical, particularly if the array is used as part of a clinical brainmachine interface (a "neural prosthetic"). Fee and Leonardo [14] described a motorized chronic microdrive with two movable electrodes that is suitable for freely behaving small animals such as the zebra finch. This device, which uses two miniature electric motors, was operated under human control.

There is a clear need for "smart neural implants" that can autonomously position large arrays of electrodes to optimize and maintain signal quality. The system described in this paper is a prototype for such a system, and is the first to autonomously isolate and maintain high quality neuronal signals. We describe a four electrode microdrive capable of semi-chronic operation in non-human primates. The microdrive can be affixed to a standard recording chamber for weeks at a time. Semi-chronic operation is made possible by the microdrive's compact design, achieved by a novel design using piezoelectric linear actuators. We also describe an algorithm for autonomously positioning electrodes to isolate and maintain high-quality action potential recordings. Together the microdrive and algorithm are an effective acute recording system, autonomously acquiring high-quality neural signal on multiple electrodes, and are also a step towards fully chronic smart neural implants, the full realization of which will require MEMS miniaturization.

\section{B. Design and Control Issues}

The design of a semi-chronic microdrive presents several issues. First, the overall device must be of minimal size and weight so that it does not significantly affect behavior in awake animal subjects, and can be implanted semi-chronically (for several days or weeks at a time). Many commercially available motorized microdrives use relatively large actuators and are meant only for acute experiments.

Miniature actuators often have very small force output, and require special attention to minimize losses in power from, for example, friction due to misalignment. High precision movement is necessary to obtain optimal signal quality, given that action potentials from a typical cell can be lost by movements as small as 50 microns. Gears and lead screws, which are commonly used, can often introduce a significant amount of imprecision in the drive due to gearing backlash. A relatively long stroke is also needed, since a range of motion of several millimeters, if not

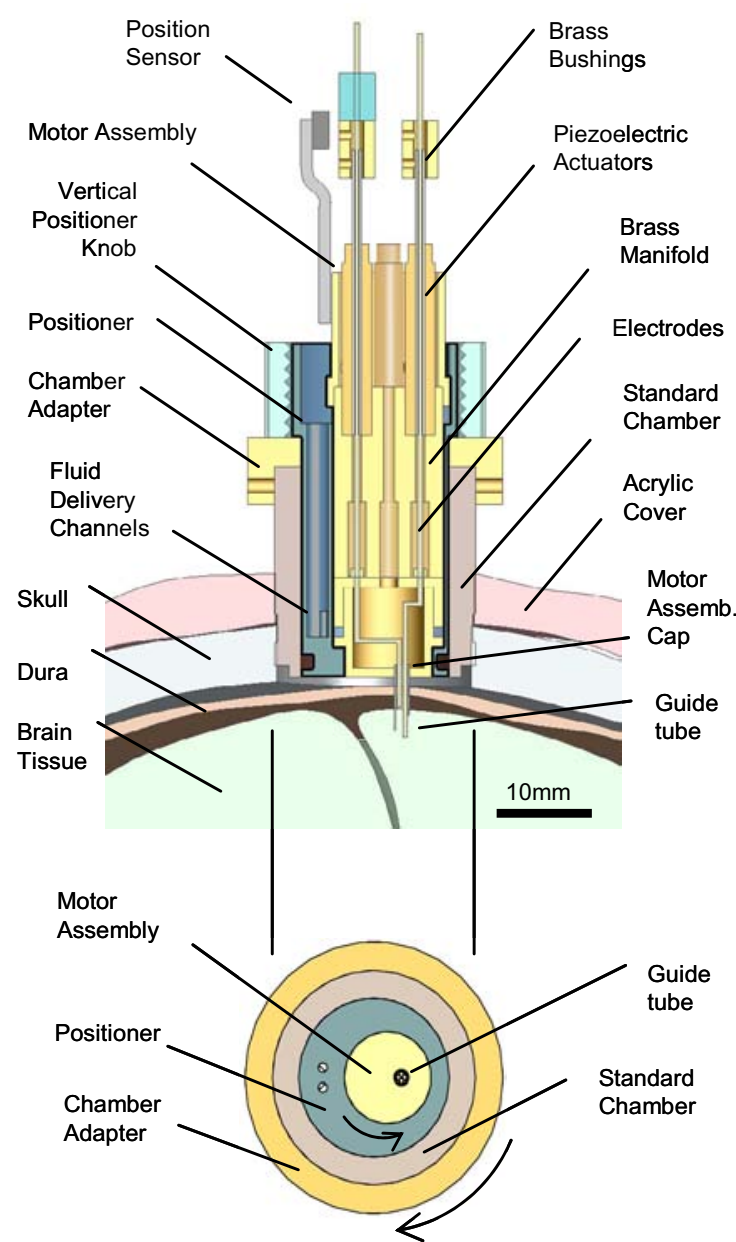

Figure 2. Cross-section of the microdrive inside of the chamber, illustrating relative position to skull and brain tissue, and front view of the device: rotation of the motor assembly and positioner allows XY positioning of the guide tube.

centimeters, is often required depending on the depth of the target structure, and the accuracy of the implantation procedure. The microdrive must also be able to keep the electrodes stable while subjected to significant stresses and vibrations from the freely moving animal.

Controlling a microdrive to isolate and maintain action potentials from active neurons in vivo is a difficult task even for experienced experimentalists. An autonomous control algorithm must not only discriminate and optimize action potentials in signals with significant noise levels, but must also deal with eventualities such as the presence of multiple cells, dying cells, cells with low firing rates and transient noise artifacts due to subject movements. Several tasks that are normally accomplished with human input must be done in an unsupervised manner, including setting a threshold for detection of action potentials and determining the number of distinct neurons in a recording.

\section{SUMmARY OF THE ROBOT DESIGN}

\section{A. Motorized microdrive design}

The basic design of our prototype is shown in Figure 1. The prototype is designed to fit inside a standard laboratory cranial chamber, used for acute experiments in non-human primates. This allows for semi-chronic operation, in which the microdrive remains in the chamber for up to several 


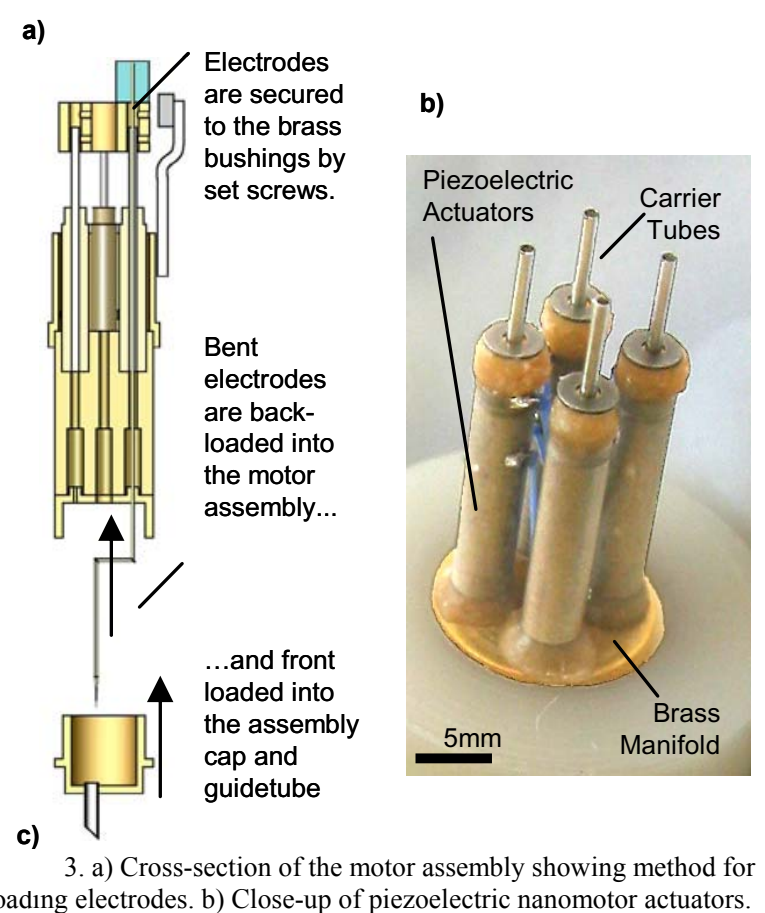

weeks at a time. A semi-chronic design has the advantage that the device can be repositioned over a different region with minimal effort and without need for additional surgeries.

As illustrated in Figure 2, the device consists of a core motor assembly that fits inside a gross vertical and horizontal positioner. The positioner in turn is fitted inside the chamber through a chamber adapter. The motor assembly consists of four piezoelectric actuators, a cylindrical brass manifold and a cylindrical cap, as shown in the figure. A short guide tube emerges from the cap, with four inner channels spaced 500 microns apart through which the electrodes are lowered. This guide tube is off-center relative to the motor assembly, while the motor assembly and positioner are arranged as non-concentric cylinders. Rotation of both the motor assembly and the positioner adjusts the horizontal or X-Y position of the guidetube relative to the chamber over an $6 \mathrm{~mm}$ diameter area, as shown in Figure 2. The positioner is constrained to move only in the vertical direction relative to the chamber adapter by two slots on its sides that match two set screws on the adapter. This allows gross vertical or $\mathrm{Z}$ positioning of the electrodes by turning a knob that engages the outside threads at the top of the positioner, as shown in Figure 2. Once the horizontal location of the guidetube has been determined and the device placed inside the chamber and lowered vertically by the positioner knob, set screws lock all the parts together, and the electrodes are then advanced by the actuators.

The electrodes are positioned by custom-made "Nanomotor" piezoelectric linear actuators (Klocke Nanotechnik, Germany, part NMSB0T10). The actuators, shown in Figure $3 \mathrm{~b}$, are $3 \mathrm{~mm}$ in diameter and $22.5 \mathrm{~mm}$ in length. These actuators were chosen for their accuracy (unloaded, they can be positioned with nanometer accuracy), high range of motion $(5 \mathrm{~mm})$, relatively high force output

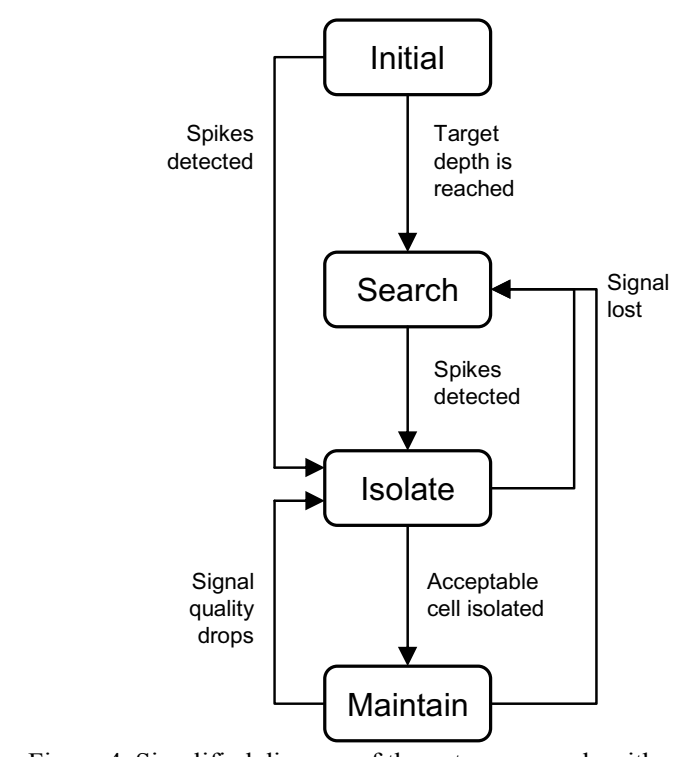

Figure 4. Simplified diagram of the autonomous algorithm state machine.

(up to $0.03 \mathrm{~N}$ of force), and direct linear drive (no gears or lead screws are needed to convert rotary to linear motion), thereby avoiding inaccuracies in positioning due to gearing backlash. The actuators are activated by a sawtooth-shaped voltage signal with a minimum peak-to-peak amplitude of $30 \mathrm{~V}$. The frequency and amplitude of the sawtooth wave determine the speed of positioning (maximum of approximately $2 \mathrm{~mm} / \mathrm{s}$ ). The piezoelectric drives are mounted on a brass manifold that helps absorb the reaction forces that occur during activation of the drives. This prevents the motion of each drive from affecting the position of the others.

The piezoelectric element in each actuator drives a hollow steel carrier tube through its center. Each electrode is passed through the center of one of these tubes and attached at the top to small brass bushings by set screws. The electrodes (FHC Inc., USA, part UE-RA1), are Pt-Ir wires (125 micron diameter), sharpened and glass coated a length of $5 \mathrm{~mm}$ at the tip, and insulated by .008 " OD polyimide tubing the rest of the length, with $10 \mathrm{~mm}$ exposed at the end for electrical connection. Typical impedance is 1.5 to 3 MOhms at $1 \mathrm{kHz}$. Each electrode is placed inside a $27 \mathrm{ga}$ steel tube, and the tube is pre-bent by 90 degrees in two locations such that one end is aligned with the actuator, and the sharpened end is aligned with the guide tube, as shown in Figure 3a. As its name suggests, the guide tube constrains the electrodes' lateral movements. Its sharpened tip also helps to penetrate the tough dural membrane surrounding the brain. The guide tube is made from hypodermic steel tubing (0.051" ID diameter) cut to size with four 0.012" ID .016" OD polyimide tubes arranged inside (see Figure 2b).

A position sensor, shown in Figure 3a, was mounted on the device to track the movement of one of the electrodes for calibration and initial testing. The position sensor consists of a Hall-effect magnetic field sensor microchip (Micronas GmbH, Germany, part HAL401) and a small magnet attached to the brass bushing of the electrode drive. The output voltage of the sensor is proportional to its position relative to the magnet. The sensor output was 


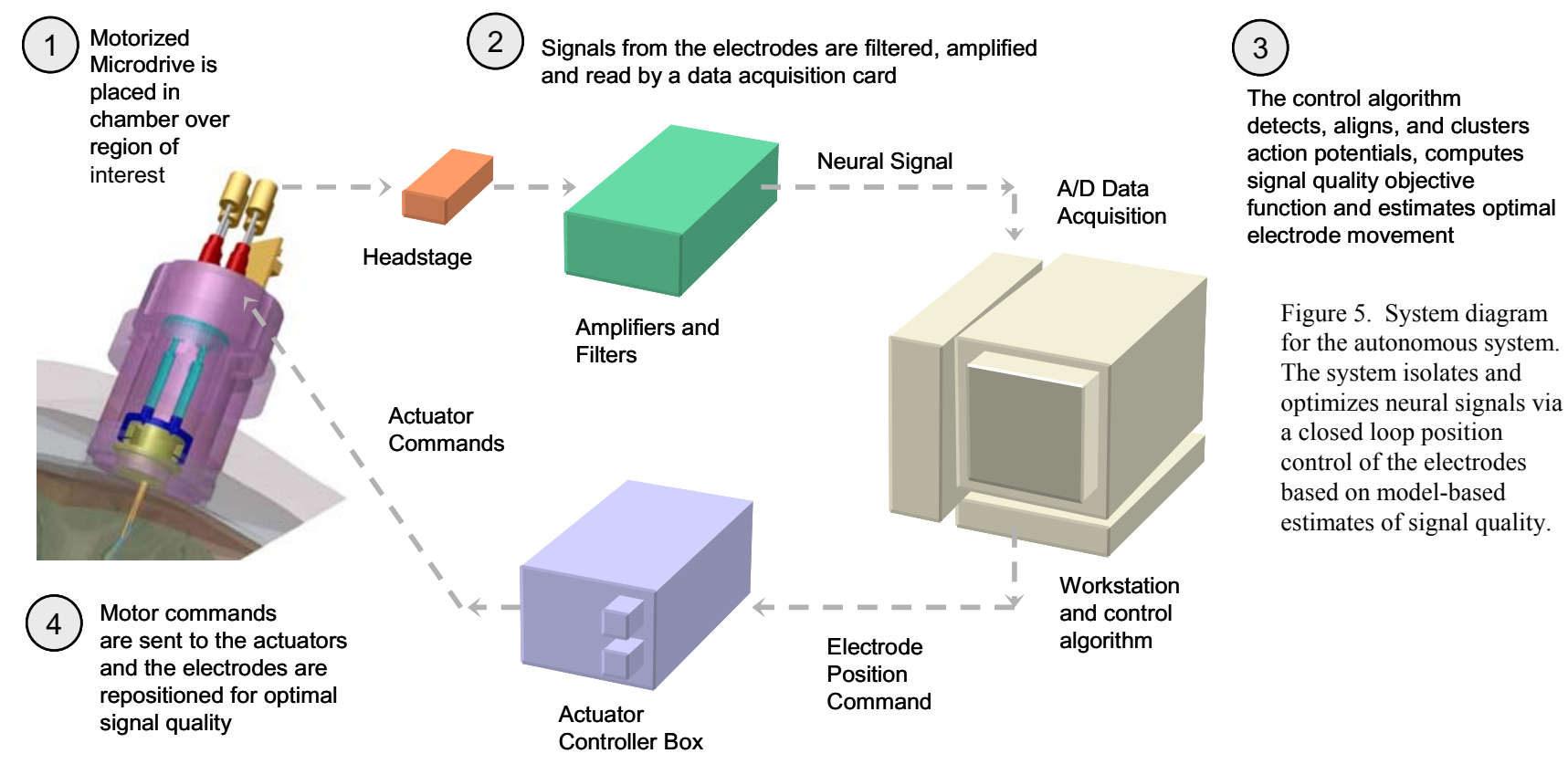

measured with a data acquisition card (National Instruments, USA). The resolution of the card allowed sensing changes in position of one micron over a range of $5 \mathrm{~mm}$.

\section{B. Microdrive fabrication and preparation}

The chamber adapter, positioner, turning knob and parts of the motor assembly were machined from Ultem polyethermide (McMaster Carr Supply Co., part 8686K76). This material matches the chamber material, and exhibits high temperature and chemical resistance, biocompatibility and machinability properties. Figure 1 shows the final prototype device. The overall device weighs approximately $40 \mathrm{~g}$.

Movement of the piezoelectric microdrives was characterized and calibrated by observing and measuring its motion under a standard microscope. The actuators move approximately one micron per commanded step. This movement was tested in free air and in gelatin, and finally in animal cortex, showing only small variations (approximately 10\%) in step size.

\section{SUMMARY OF THE CONTROL ALgORITHM}

The basic architecture of the autonomous control algorithm for one electrode consists of two layers. The first (outer) layer is a state machine, which performs the initial search for action potentials, monitors the cell isolation and maintenance processes, and commands appropriate actions for the eventualities mentioned above. The second (inner) layer is the stochastic optimization method developed in Nenadic and Burdick (2004). This method optimizes signal quality in the presence of action potentials, given that only noisy observations are available.

A simplified diagram of the state machine is shown in Figure 4. At each state, the algorithm samples the neural signal for a short length of time (in this case, $20 \mathrm{sec}$ ) and searches for action potentials. Depending on the outcome, the state machine may execute a change of state and/or send a move command to the microdrive to reposition the electrode.
The system is started in the "Initial" state, once the microdrive device has been positioned over the desired recording region inside the chamber. Electrodes are advanced 250 microns (at a velocity of 4 microns per second) between samples until one of the following events occur: either a previously determined target depth is reached, which can be obtained from anatomical data such as MRI scans [15], or until action potentials are detected in the neural signal.

Action potentials are detected using the unsupervised wavelet-based detection method presented in [16]. Traditional methods such as amplitude and power thresholding, window discrimination and matched filtering require human supervision and experience, as they depend on the amplitude, shape and phase of the action potentials recorded, which can change as the electrode moves relative to the cell body. Action potentials are considered to be present only when the number of events detected by the method exceeds a minimum firing rate, in this case $2 \mathrm{~Hz}$. Once detected, action potentials are aligned and clustered using the correlation method and finite mixture model clustering method described in [16].

If the target depth is reached without detection of action potentials, the algorithm switches to the "Search" state, which advances the electrode only 50 microns (at 4 microns per second) between samples. If action potentials are detected while in the "Initial" or "Search" states, the system switches to the "Isolate" state.

The goal of the "Isolate" state is to reposition the electrode to maximize signal quality. In the method of [16], this goal is mathematically formalized by defining a (nonnegative) objective function over a segment of a real line in the neighborhood of the cell (since the electrode is constrained to linear motion). The resulting curve is called the "cell isolation curve", and the goal is to find the position that maximizes it. The method is independent of the exact choice of objective function, but it must capture some measure of signal quality. In this paper, we test the use of two different metrics, though others are certainly possible. 
The first metric tested is the peak-to-peak amplitude (PTPA) of the recorded action potentials. The second metric is the distance in principal component space (DPCS) between a useful cell and confounding cells or noise, which may be useful in the presence of multiple spiking neurons.

Since only noisy observations of the objective function are available, the objective is defined as a regression function,

$$
M(x)=E(y \mid x)
$$

where $\mathrm{y}$ is the chosen measure of signal quality, $\mathrm{x}$ is the position of the electrode along its range of motion and $E($.) is the expectation operator. We estimate the regression function with a set of polynomial basis functions, using all (or a subset of) the previous observations obtained at the preceding electrode positions. The order of the model is adaptively chosen through Bayesian theory.

Initially, the "Isolate" state moves the electrode in constant increments (in our case, 20 microns) between samples of the neural signal to collect initial samples for the basis function approximation. Once a significant regression curve is estimated, the electrode is moved in steps to the maximum of the curve, which is updated with each new observation. The state machine remains in the "Isolate" state until either an upper bound on signal quality is reached (which prevents the algorithm from driving the electrode into the body of a cell that lies directly in its path) or it is determined that the maximum of the regression function has been reached. The algorithm is determined to have reached the maximum of the regression function when the commanded step size reaches a minimum value, in this case 1 micron, which will occur when the gradient approaches zero. If the maximum value that is realized is below a lower

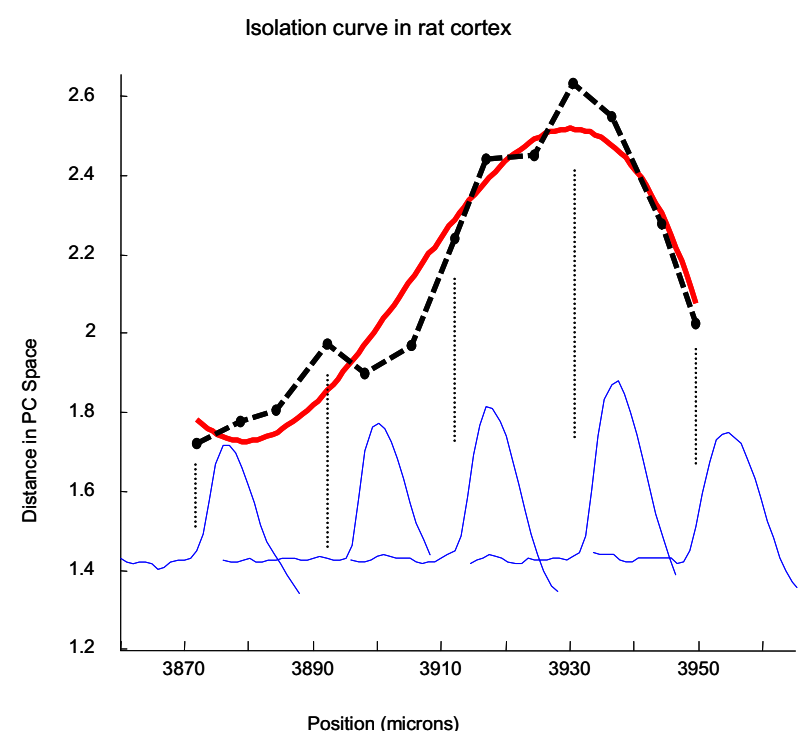

Figure 6. Isolation curve recorded from rat cortex. The algorithm seeks the maximum of such a curve to optimize the neural signal. The dashed line shows the measured signal quality using the DPCS metric (see text) as a function of electrode position. Average recorded spike shapes are plotted in blue for five representative positions. Each spike shape is associated with a particular position by a dotted line (i.e. the leftmost spike shape is that observed at position 3872 microns). The solid red line shows the fitted basis function approximation calculated after all positions were sampled. bound of signal quality, then the cell isolation is considered unacceptable, and the algorithm switches back to the "Search" state.

If the cell is considered isolated, the state machine changes to the "Maintain" state. In this state, the algorithm samples the neural signal while keeping the electrode stationary. This continues until the measured signal quality falls below the acceptable SNR level. Once this occurs, the cell is no longer considered isolated, and the algorithm switches back to the "Search" state in order to re-acquire the signal.

\section{EXPERIMENTAL SETUP AND PROCEDURE}

Initial experiments were performed on anesthetized rats to test the basic operation of the microdrive and control algorithm. Single-electrode experiments were conducted in the posterior parietal cortex [17] of an awake, behaving adult macaque monkey. The microdrive was installed in the cranial chamber at the beginning of each recording session. Using the vertical positioning knob, the device was lowered by hand to a target depth. The algorithm was then activated and the system operated autonomously. The monkey performed simple saccade (eye movement) tasks in a darkened room. The animal care and handling in these experiments were in accord with the guidelines of the National Institutes of Health and have been reviewed and approved by the local Institutional Animal Care and Use Committee.

A diagram of the autonomous system is shown in Figure 5. The output of the electrode from the microdrive was connected to a DAM-80 (World Precision Instruments Inc., USA) headstage and amplifier in the rat experiments, and to a Plexon (Plexon Inc., USA) headstage and amplifier in the monkey experiments. The signal output from the amplifiers in both setups was recorded by a data acquisition card (National Instruments Inc., USA). Filter and gain settings varied with experimental conditions and objectives. The piezoelectric motors were powered and activated by a controller purchased with the actuators (Klocke Nanotechnik, Germany, part NWC). The control algorithm was implemented in Matlab (Mathworks Inc., USA).

\section{EXPERIMENTAL RESULTS}

The motorized microdrive in single-electrode configuration and the control algorithm were used to record from neurons in rat and monkey cortex. Figure 6 shows sample results from rat cortex. Shown in the figure is signal quality (in this case measured by the DPCS metric) as a function of electrode position, with the corresponding averaged spike shapes at selected positions. These results demonstrate the presence of our conceptual isolation curves in rat cortex, which are the basis of our autonomous algorithm.

Figure 7 shows a sample result of autonomous cell isolation in monkey cortex. In this case, the algorithm was initiated after the microdrive was installed in the chamber and allowed to operate autonomously without human intervention. The algorithm first advanced the electrode in the "Initial" and "Search" states for over $1.5 \mathrm{~mm}$ until faint spike activity was detected. Shown in Figure $7 \mathrm{a}$ is the sequence of steps taken by the algorithm once the state 

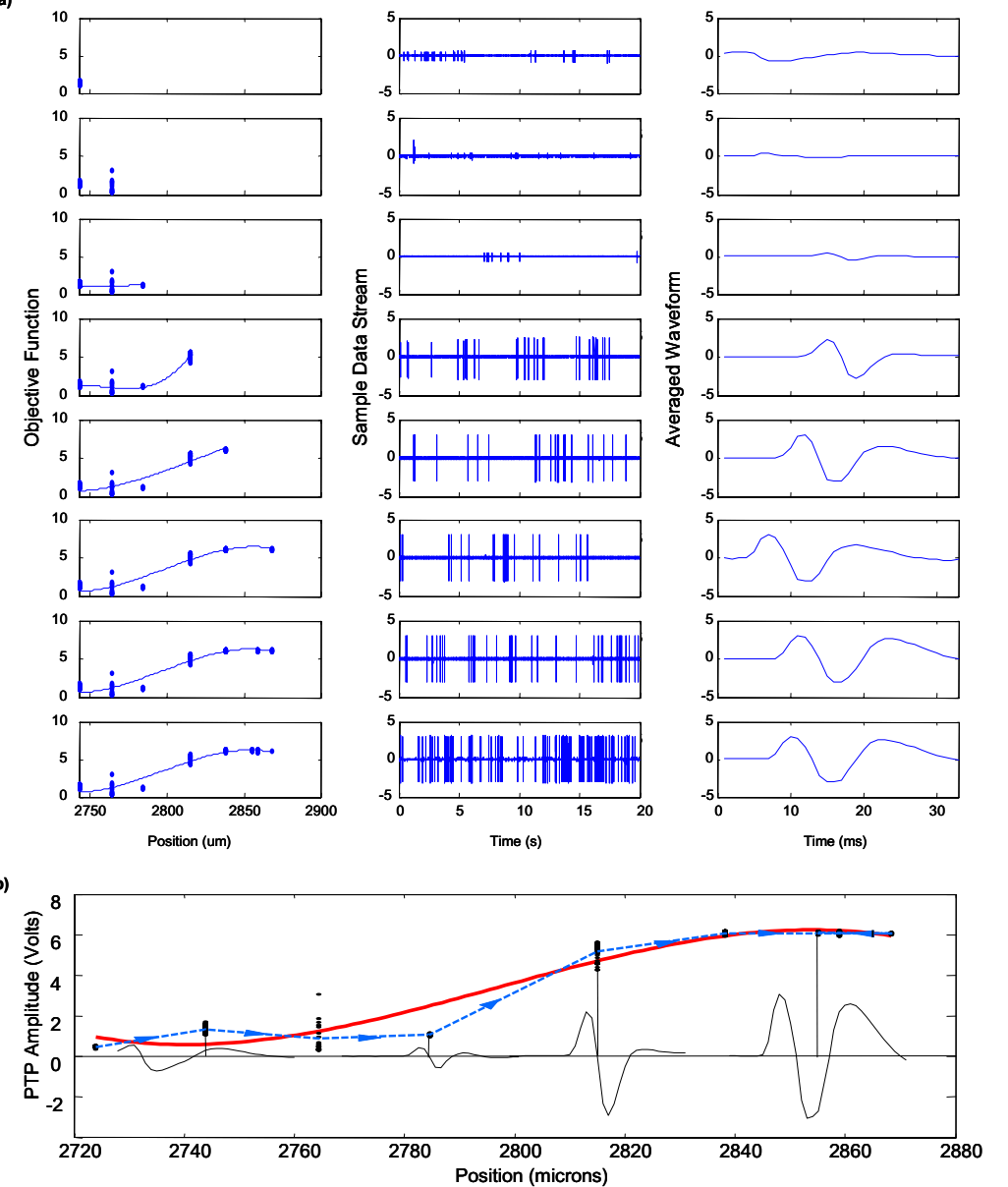
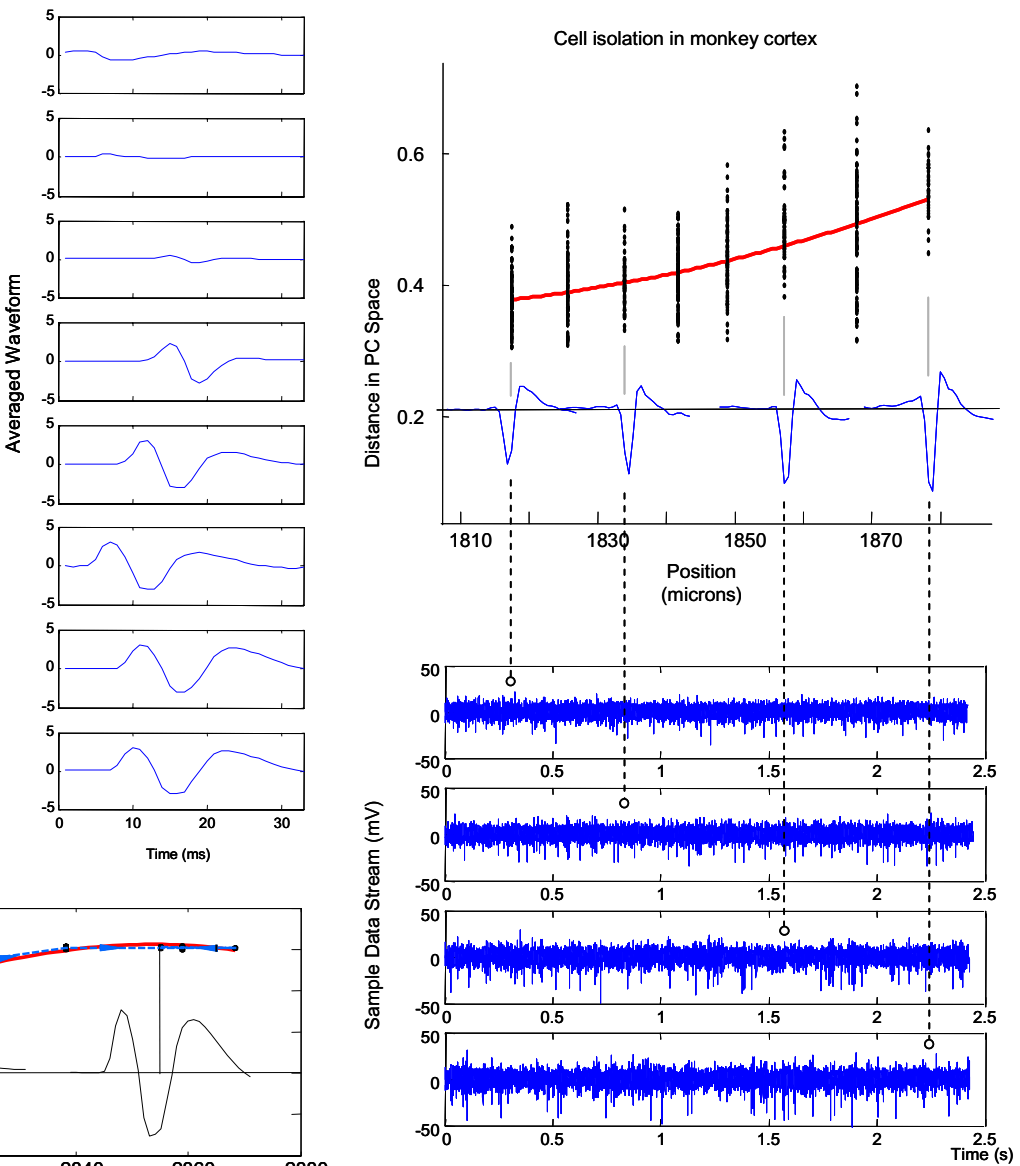

Figure 7. Cell isolated in monkey cortex using the autonomous semi-chronic microdrive system. a) Progression of algorithm in presence of action potentials. The left column shows snapshots of the sampled objective function and the basis function approximation; the middle column shows the signal spike train; the right column shows the averaged waveform of the detected spikes. b) Final isolation curve and average spike waveforms at each position. In this case, the signal was optimized until the maximum of the isolation curve was found. c) In this case, the cell was considered to be isolated when the signal quality reached a maximum value, in order to avoid potential damage to the cell. The top plot shows the sampled signal quality (in black) and the fitted regression function (in red) as a function of electrode position, with average action potentials shown for four different positions. The data streams that correspond to the four positions are shown below (in blue), with correspondence indicated by the dashed lines.

machine transitioned to the "Isolate" state. The first column shows the positions of the electrode and the measured objective function (in this case, PTPA). The second column shows the corresponding data stream at that position, and the third column shows the average spike waveform. As shown, the algorithm first advanced the electrode in constant increments. After enough observations were made to allow an adequate model to be fitted, the polynomial fit was made, shown as a solid line. Once a peak in the objective function was detected, the control algorithm repositioned the electrode toward the optimal location. The sequence ended when the cell was determined to be isolated by the algorithm, as per the minimum step size criteria previously discussed. Figure $7 \mathrm{~b}$ is a concatenated plot of the sequence, showing the final isolation curve approximation (solid line), the progression of the algorithm (dotted line) and the average waveforms.

The results from Figure 7a illustrate the potential risk of unconstrained signal maximization. As shown in the second column of figure $7 \mathrm{a}$, the firing rate of the cell increased as the electrode moved forward, indicating that the electrode was affecting cell behavior possibly due to very close proximity. Figure $7 \mathrm{c}$ shows final results of the algorithm in monkey cortex in which the upper bound on signal quality was implemented in order to prevent potential damage to the cell. In this case, the algorithm advanced the electrode to maximize the regression function until the upper bound was reached, at which point the cell was considered isolated.

To date, several dozen cells have been isolated with the autonomous system in monkey cortex. Cells have been isolated and maintained for up to 3 hours. Figure 8 shows a sample time history of signal quality after the cell has been determined isolated by the algorithm and the state machine entered the "Maintain" state. As shown, signal quality (measured by the SNR) degrades over time, possibly due to tissue migration. Once the signal dropped below the lower bound SNR threshold, the algorithm automatically reinitiated the search and isolation processes and reacquired the signal. The continuous measurements of the PTPA metric shown in the figure and the consistency of the spike shape provide some evidence that the same cell was being tracked throughout the entire experimental session.

\section{CONCLUSIONS}

The novel miniature motorized microdrive was shown capable of advancing and retracting electrodes in cortical 


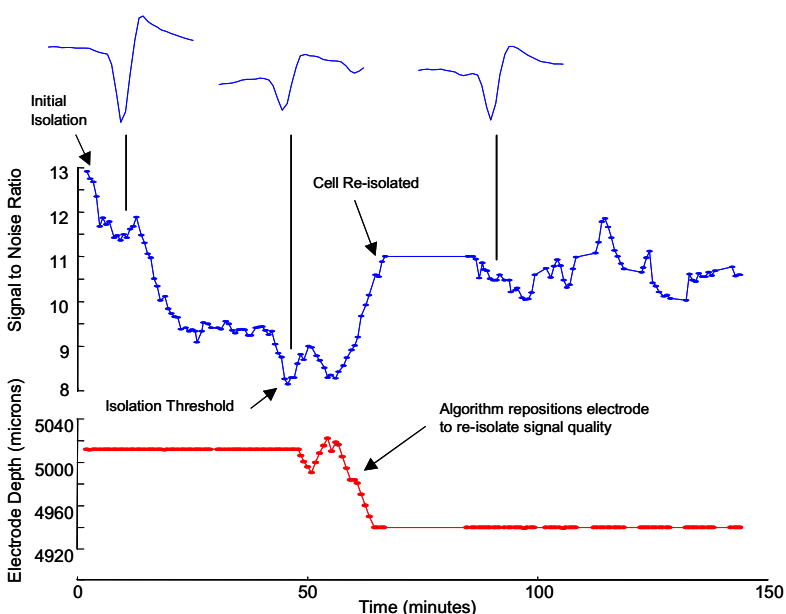

Figure 8. Time history of signal quality over a period of 2.5 hours, after the cell was isolated by the autonomous algorithm. The figure shows how signal quality slowly degraded, perhaps due to tissue migration. Once signal quality degraded below a minimum threshold, the autonomous algorithm repositioned the electrode to reacquire an acceptable signal quality level.

tissue with micron precision and recording high-quality neural signals. The electro-mechanical design of the microdrive addresses several issues in recording implant design. The microdrive performed well over several dozen recording sessions without sign of performance degradation. The device is rugged in construction, safe and relatively easy to install in the head chamber and to reload and maintain electrodes.

The algorithm presented in this paper was shown to autonomously command the microdrive to seek and isolate action potentials from cells. All of the different methods used in the isolation algorithm, from spike detection, alignment and clustering to regression function model selection and estimation, require no supervision and account for the stochastic nature of the task. The results shown for monkey cortex were obtained with no human intervention once the microdrive was placed inside the chamber.

The novel microdrive and the algorithm presented here do not necessarily need to be implemented together. The microdrive design provides a working device for acute or semi-chronic recordings that can be controlled by a human operator, or by an alternate control algorithm. Similarly, the algorithm can be used to control other microdrives for autonomous operation. The successful integration of the two systems, however, is presented as a first step towards future "smart" neural implants that are fully autonomous. Such autonomous implants could contribute to the efficiency and flexibility of neurophysiological studies by freeing the experimentalist from time-consuming tasks such as frequent implantation surgeries and finding and maintaining highquality neural signals.

\section{ACKNOWLEDGMENT}

We thank the members of the Andersen lab at Caltech, especially Bradley Greger, Daniella Meeker, Bijan Pesaran, Boris Breznen, Sam Musallam, Kelsie Pejsa and Lea Martel. Thanks also to Aimee Eddins and Rodney Rojas for help in fabricating the prototype.

\section{REFERENCES}

[1] Porada I, Bondar I, Spatz WB and Kruger J. "Rabbit and monkey visual cortex: more than a year of recording with up to 64 microelectrodes," $J$ Neurosci Methods 95: 13-28, 2000.

[2] Williams JC, Rennaker RL and Kipke DR. "Long-term neural recording characteristics of wire microelectrode arrays implanted in cerebral cortex," Brain Research Protocols 4: 303-313, 1999.

[3] Rousche PJ and Normann RA. Chronic recording capability of the Utah intracortical electrode array in cat sensory cortex," J. Neurosci. Meth., 82:115 (1998).

[4] Gray CM, Maldonado PE, Wilson M and McNaughton B. "Tetrodes markedly improve the reliability and yield of multiple single-unit isolation from multi-unit recordings in cat striate cortex," J Neuroscience Methods 63: 43-54.

[5] Wall PD, Freeman J, Major D. "Dorsal horn cells in spinal and in freely moving rats," Exp Neurol 19: 519-529, 1967.

[6] Kubie JL. "A Driveable bundle of microwires for collecting single-unit data from freely-moving rats," Physiology \& Behavior 32: 115-118, 1984.

[7] Vos BP, Wijnants M, Taeymans S and De Schutter E. "Miniature carrier with six independently moveable electrodes for recording of multiple single-units in the cerebellar cortex of awake rats," J Neurosci Methods 94: 19-26, 1999.

[8] Venkatachalam S, Fee MS and Kleinfeld D. "Ultra-miniature headstage with 6-channel drive and vacuum-assisted micro-wire implantation for chronic recording from the neocortex," J Neurosci Methods 90: 37-46, 1999.

[9] Kralik JD, Dimitrov DF, Krupa DJ, Katz DB, Cohen D and Nicolelis

MAL. "Techniques for long-term multisite neuronal ensemble recordings in behaving animals," Methods 25: 121-50, 2001.

[10] Tolias AS, Siapas AG, Smirnakis SM, Logothetis NK. "Coding visual information at the level of populations of neurons," Program No. 557.5. 2002 Abstract Viewer/Itinerary Planner. Washington, DC: Society for Neuroscience, 2002.

[11] Keating JG and Gerstein GL. "A chronic multi-electrode microdrive for small animals," J Neurosci Methods 117: 201-206, 2002.

[12] Hoffman KL and McNaughton BL. "Coordinated reactivation of distributed memory traces in primate neocortex," Science 297: 2070-2073, 2002 .

[13] deCharms RC, Blake DT and Merzenich MM. "A multielectrode implant device for the cerebral cortex," J Neurosci Methods, 93: 27-35, 1999.

[14] Fee MS and Leonardo A. "Miniature motorized microdrive and commutator system for chronic neural recording in small animals," $J$ Neurosci Methods 112: 83-94, 2001.

[15] Scherberger H, Fineman I, Musallam S, Dubowitz DJ, Bernheim KA, Pesaran B, Corneil BD, Gilliken B, and Andersen RA. "Magnetic resonance image-guided implantation of chronic recording electrodes in the macaque intraparietal sulcus," J Neurosci Methods 130:1-8, 2003

[16] Nenadic Z, Burdick JW. "Spike detection using the continuous wavelet transform," IEEE Trans Biomedical Eng vol. 5, pp. 74-87, 2004.

[17] Andersen RA and Buneo CA. "Intentional maps in posterior parietal cortex," Ann.Rev. Neurosci 25: 189-220, 2002. 